\title{
Prevalence of geophagia and its contributing factors among pregnant women at Dr. George Mukhari Academic Hospital, Pretoria.
}

\author{
L.R.Macheka ${ }^{1}$, J.O.Olowoyo ${ }^{1}$, L.Matsela ${ }^{2}$, A.A.Khine ${ }^{3}$
}

1. Sefako Makgatho Health Sciences University, Biology

2. Sefako Makgato Health Sciences University, Obstetrics and Gynaecology

3. Sefako Makgato Health Sciences University, Chemical Pathology

\begin{abstract}
:
Introduction: Geophagia is very common among pregnant women, particularly in Africa. There are many reasons given for geophagia such as cultural, medicinal and religious, making it an acceptable norm regardless of health risks involved.

Objectives: The study explored prevalence and factors influencing geophagia among women visiting an antenatal clinic in Pretoria.

Methods: A quantitative survey was done on a convenience sample of 597 pregnant women and structured interviews conducted. Statistical analysis was done using simple percentage and interview data analyzed using Epi Info statistical software.

Results: Geophagia was reported by $54.0 \%$ of the women $(n=323)$ and of these, $75.2 \%(n=243)$ ate at least 3 teaspoons per day. Reasons for the practice ranged from simple unexplained craving to belief that soil acts as an iron supplement. The study revealed that education levels did not act as a contributing factor as both literate and illiterate women were consumers. Partners of consumers played a key role in influencing the practice as most consumers were not married.

Conclusion: Geophagia is practiced by a considerable proportion of pregnant women in this area. Greater vigilance may be needed as part of the antenatal classes to avoid potentially harmful effects of the habit.

Keywords: Geophagia, pregnant women, Dr. George Mukhari Academic Hospital, Pretoria.

DOI: http://dx.doi.org/10.4314/ahs.v16i4.13

Cite as: Macheka LR, Olowoyo JO, Matsela L, Khine AA. Prevalence of geophagia and its contributing factors among pregnant women at Dr. George Mukhari Academic Hospital, Pretoria. Afri Health Sci 2016;16(4): 972-978. http:/ / dx.doi.org/10.4314/ abs.v16i4.13
\end{abstract}

\section{Introduction}

Geophagia is defined as the deliberate consumption of earth in the form of soil or clay by both humans and animals and has been described as a complex eating behaviour ${ }^{1,2}$. Although this practice has a long old tradition, dating back to the $18^{\text {th }}$ century physicians who first classified it as a form of pica $^{2,3}$, many academic researchers have often used adjectives such as odd, strange, curious and perverted when describing the habit. Geophagia is universal in distribution and most indications of its origins have been found in Africa, the earliest evidence having originated from a location close to Kalambo Falls at the boarder of Tanzania and Zambia ${ }^{4,5 .}$ From there, it is believed to have spread to other parts of the world where it is prevalent today through slavery ${ }^{5}$. There are many reasons given for geophagia such as cultural, medicinal,

\section{Corresponding author:}

L.R. Macheka,

Sefako Makgatho Health Sciences University, Biology, Email: misslindamacheka@gmail.com religious and mineral deficiency, making it an acceptable norm, regardless of the health risks involved.

In earlier times, soil was purported to have a soothing effect if used as a drug and could also be used as a remedy for ulcers, diarrhoea and menstrual pain if administered orally, rectally or as an enema ${ }^{6}$. Several studies have led to the hypothesis that animals ingest soil or clay unconsciously to alleviate gastrointestinal distress as well as a way of attempting to replenish a depleted supply of certain minerals in their body ${ }^{7,8,9}$.

In this way, they parade an ability to regulate their diets to accomplish nutritional balance ${ }^{10}$. The habit of eating clay by animals in the wild for the detoxification of the body and easing of gastrointestinal infections may have accounted for the practice among humans ${ }^{11}$. Clay has also been used medicinally over the centuries for various ailments. The ancient Egyptians and Mesopotamians used it to plaster wounds and consumed it to treat various infirmities, especially those related to the gut ${ }^{12}$. Culturally, in some societies, such as the Tiv tribe of Nigeria, a craving for dirt is used as an early sign of pregnancy ${ }^{9}$. In other 
instances, geophagia is simply practiced due to the sincere pleasure of the habit. Geophagic soils include soils from gardens, mountains, river banks, termite mounts, mole hills, pits as well as earth worm casts ${ }^{13}$. Preliminary studies have also established that the mainconsumers are pregnant women and the continuous intake of the soil may pose a great health threat to them as well as to the unborn baby ${ }^{2,14,15}$. The present study therefore aims to establish the prevalence of geophagia among pregnant women attending the ante natal clinic at Dr. George Mukhari Academic Hospital, and to investigate underlying factors associated with the practice.

\section{Methods}

The study was carried out in the ante natal clinic of Dr. George Mukhari Academic Hospital, which is situated about $20 \mathrm{~km}$ north of Pretoria and lies on the border of Gauteng and North Western Province. The Academic Hospital runs daily ante natal classes with an average of three hundred cases monthly. Only pregnant women in their third trimester who had $\geq 27$ weeks' gestation and attended the antenatal clinic in Dr. George Mukhari Academic Hospital during the study period were asked to participate as the study population. A sample size of at least 288 pregnant women was requisite to achieve a $95 \%$ confidence interval (CI) with a power of $90 \%$, presuming a non-response rate of $20 \% 16$. A total 610 pregnant women were interviewed in total, although 13 were excluded because of incomplete questionnaires, leaving the ultimate study sample at a figure of 597. The sampling bias was reduced by inclusion of all pregnant women who are $>27$ weeks of gestation and are geophagic so as to ascertain that geophagia had adequately taken place since the beginning of pregnancy. Women less than 27 weeks were excluded and the exclusion criteria assisted with validity of the data as it screened out non - specific findings or confounded factors.

The study was conducted from February 2014 to November 2014. All participants signed a written consent before participation in the study. The study was conducted in accordance with the Declaration of Helsinki of 1975 and the protocol was approved by Medunsa Ethical and Research Committee (MREC/P/254/2013: PG) before commencement. Interviews were conducted by administering open ended questionnaires to pregnant women attending the ante natal clinic at Dr. George Mukhari Academic Hospital. The interviews were conducted in a closed room (Room 1) after the nurse in charge explained to the pregnant women the objectives of the study and asked those willing to participate to follow the interviewer individually to the interview room. Questionnaires were used to collect data on participant demographics, earth eating practices and the reasons for eating the soil. These questionnaires were administered verbally by the interviewer between $7.30 \mathrm{am}$ and mid - day after the pregnant women get their health awareness classes from the nurses. The interviews were carried out every day from Monday to Thursday when the antenatal clinic was open. Prevalence was determined by simple percentage, andinterview data was analysed using Epi Info statistical software (version 7). Confidence Intervals (95\% CI) were calculated using logistic regression in order to forecast socio demographic characteristics and a p-value of $<0.001$ was regarded as significant.

\section{Results}

\section{Prevalence of geophagia}

Of the 597 participants interviewed, 323 indicated that they practiced geophagia, representing $54.0 \%$ of the study population. The age of consumers ranged between 15 and 44 years, with a mean age of 29 years and a standard deviation value of 6.8 (Table 1).

Table 1: Table showing the marital status of soil consumers

\begin{tabular}{|l|r|r|r|r|r|}
\hline \multicolumn{1}{|c|}{ Marital Status } & Frequency & Percent & Cum. Percent & $\mathbf{9 5 \%}$ CI Lower & 95\% CI Upper \\
\hline Single & 269 & $83.28 \%$ & $83.28 \%$ & $78.66 \%$ & $87.09 \%$ \\
\hline Married & 44 & $13.62 \%$ & $96.90 \%$ & $10.17 \%$ & $17.96 \%$ \\
\hline Divorced & 10 & $3.10 \%$ & $100.00 \%$ & $1.58 \%$ & $5.80 \%$ \\
\hline TOTAL & $\mathbf{3 2 3}$ & $\mathbf{1 0 0 . 0 0 \%}$ & $\mathbf{1 0 0 . 0 0 \%}$ & & \\
\hline
\end{tabular}

Of these consumers, $7 \%(\mathrm{n}=23)$ were between the ages of 15 and $18,19 \%(n=61)$ between 19 and 24 years, $36 \%$ $(\mathrm{n}=116)$ between 25 and 30 years, 24\% $(\mathrm{n}=78)$ between 31 and 36 years, $13 \%(n=42)$ between 37 and 42 years and only $1 \%(\mathrm{n}=3)$ above 43 years.

African Health Sciences Vol 16 Issue 4, December, 2016 


\section{Reasons for Geophagia}

Although $52.3 \%(n=169)$ of the women could not explain their cravings for soil, one of the key reasons for soil consumption among the pregnant women was the cravings due to taste, texture and smell, $(26.0 \%, \mathrm{n}=84)$ especially before the rains. A total of $9.0 \%(n=29)$ of the consumers also cited that they believed soil improved their health and that of the baby as it acted as an iron supplement. Among the health reasons, $9.0 \%(\mathrm{n}=29)$ of the consumers also mentioned that it helps with heartburn and morning sickness during pregnancy. Other reasons cited by the consumers were to simply while away time during the day $(5.6 \%, \mathrm{n}=18)$ as well as $10.5 \%(\mathrm{n}=$ 34) of the consumers citing the sheer enjoyment of the soil's salty taste as a reason for consumption. Apart from these, $3.0 \%(n=10)$ consumers pointed out that they eat soil as a supplement for another form of pica, such as eating ice, chalk or washing powder.

\section{Factors associated with Geophagia:}

\section{Marital status}

In this particular study, $83.0 \%(n=268)$ of the consum- ers were not married (Table 1), while 13.9\% $(n=45)$ were married and 3.1\% $(\mathrm{n}=10)$ were divorced. The study also revealed that $99.1 \%(\mathrm{n}=320)$ of the participants interviewed specified that their partners did not eat soil. While $86.4 \%(\mathrm{n}=279)$ of the participants' partners were not aware of the soil eating habit, the remaining 7.1\% ( $\mathrm{n}=$ 23) showed strong disapproval of the habit, $2.8 \%(n=9)$ did not mind, $2.8 \%(n=9)$ would get angry at seeing their partner eating soil and $0.9 \%(\mathrm{n}=3)$ of the partners also ate soil. In one case, a consumer said, "I only eat when my husband is not around."

\section{Family influence}

A total of $49.6 \%(n=160)$ of consumers confessed that either their mother or grandmother was geophagic, compared to $10.6 \%(n=29)$ of non - consumers who had a history of geophagia in their family. However, 16.3\% (n $=45$ ) of non - consumers had a sister who was eating soil as compared to $9.2 \%(\mathrm{n}=30)$ of consumers and this would evince that the sister of the non - consumer would have been the one to pick up the habit from the mother or grandmother.

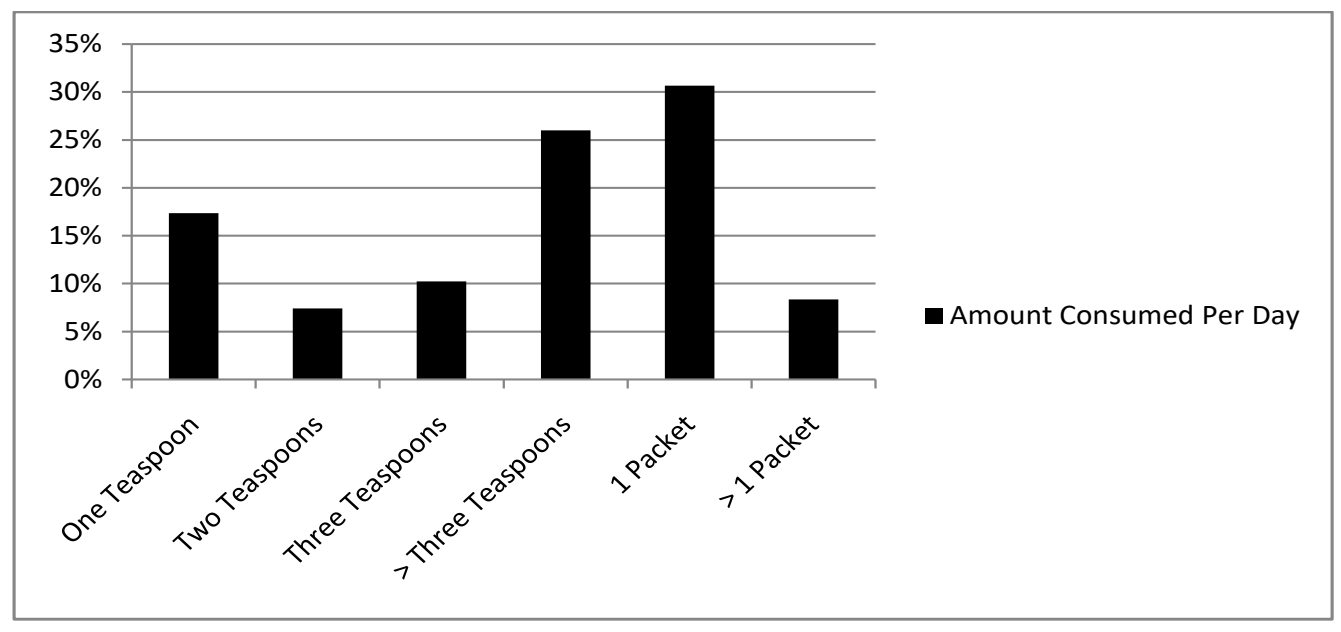

Figure 1. Figure showing amount of soil consumed per day

\section{Employment status}

Figure 2 shows that $73.4 \%(n=237)$ of consumers were unemployed, with $85.8 \%(\mathrm{n}=277)$ of them earning less than R5 000 per month. 


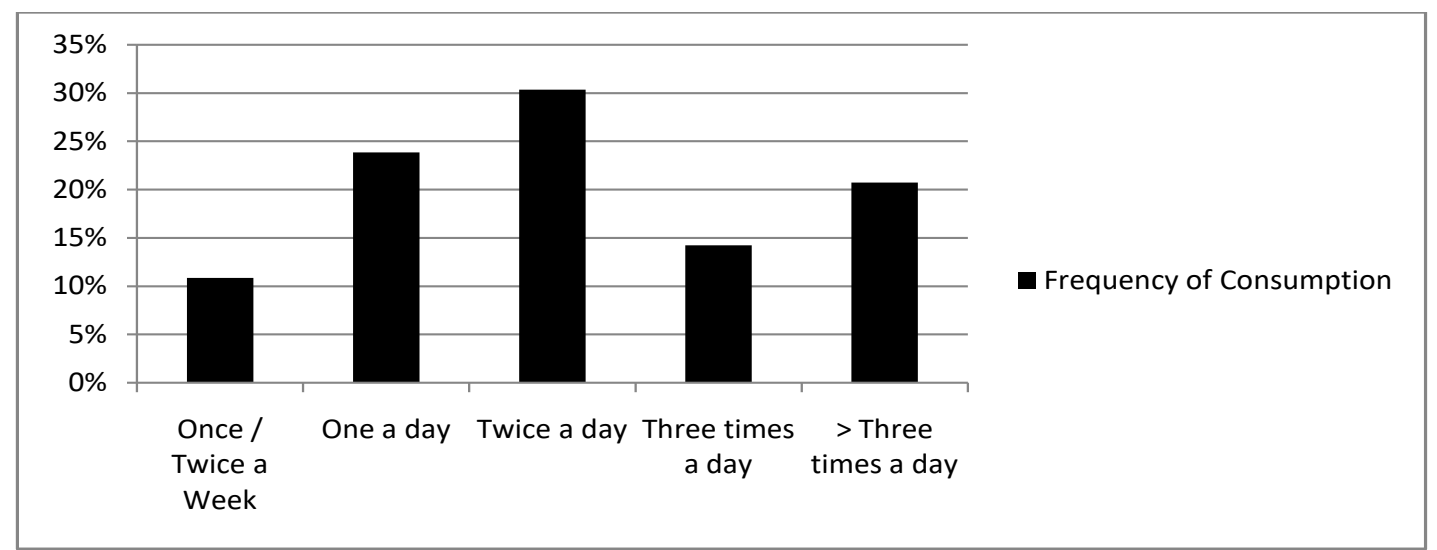

Figure 2: Figure showing frequency of consumption per day

\section{Soil eating practices:}

\section{Amounts consumed}

When asked to estimate the amount of soil that they consume in teaspoons, $75.2 \%(\mathrm{n}=243)$ of the pregnant womenrevealed that they eat three or more teaspoons per day ashighlighted in Figure 3, where each teaspoon weighed an average of $7.9 \mathrm{~g}$. Of the geophagic soils being consumed, $57.9 \%(\mathrm{n}=187)$ were bought from the market as clayblocks and therefore, consumers could more eas- ily estimate the amount of soil consumed per day in the formof whole packets weighing an average of $310 \mathrm{~g}$ per packet. In this regard, $30.7 \%(\mathrm{n}=99)$ of the consumers ate an average of $310 \mathrm{~g}$, while $8.36 \%(\mathrm{n}=27)$ at $>310 \mathrm{~g}$ per day.

Many of these consumers complained of both occasional $(35.3 \%, \mathrm{n}=114)$ or frequent $(18.3 \%, \mathrm{n}=59)$ constipation, which indicated a direct relationship between theamounts consumed and the levels of constipation.

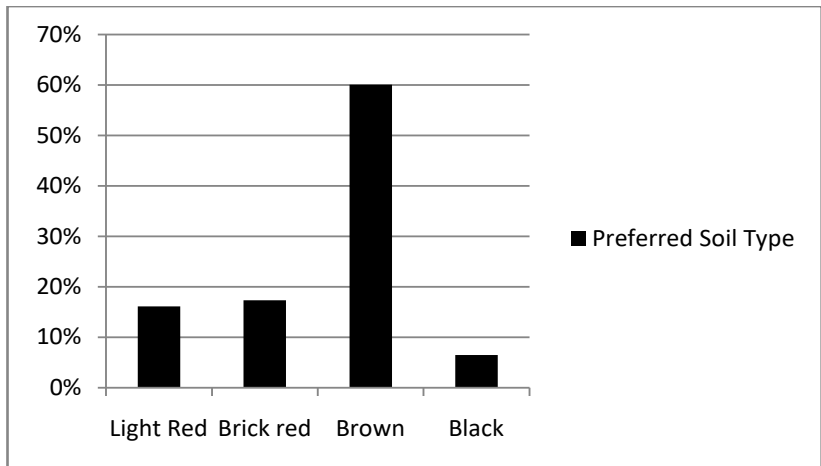

Figure 3: Figure showing the different types of soil preferred by the consumers

\section{Frequency of consumption}

According to $49.2 \%(\mathrm{n}=159)$ of the consumers, their greatest cravings for soil were experienced just after eating a meal. $35.0 \%(n=113)$ of the women ate soil three or more times a day, usually after meals. In one interview, a participant said "I don't feel that I have eaten a proper meal without eating soil afterwards." However, those that ate once a day $(23.8 \%, \mathrm{n}=77)$ usually finished the whole packet of clay in one sitting. The period of consumption widely ranged among consumers, with $26.3 \%$ ( $\mathrm{n}=85$ ) having been eating soil for as little as $1-4$ months and $18.9 \%(\mathrm{n}=61)$ for 6 months to a year. In addition, $16.7 \%$ $(\mathrm{n}=54)$ had been consumers for $1-4$ years, while $16.1 \%$ $(\mathrm{n}=52)$ ate soil for $5-10$ years. The women who claimed to have eaten soil for the longest time had been frequent consumers for more than 10 years and these accounted for $22.0 \%(n=71)$ of the total consumer population.

\section{Preferred soil type}

$60.1 \%$ ( $n=194$ ) of the consumers preferred eating the brown clay soil that was sold on the market and they denoted that it tasted nicer compared to other soils. On the other hand, the $17.3 \%(n=56)$ that preferred the brick red soil culminated to the fact that it tasted saltier than the others and they believed that it had higher iron levels making it of more nutritional value than the other soil types. Those that preferred the lighter red soil $(16.1 \%$, $\mathrm{n}=52$ ) enjoyed its taste better and said it did not stick 
to the tongue and caused less constipation. The women consuming the black soil $(6.5 \%, \mathrm{n}=21)$, however, had a notion that it had fewer microorganisms in it that could harm the baby. The black soil was mostly collected in the garden and in flower pots around theyard, which made it more accessible for the consumers. Although most of the consumers ate the soil directly from its source, $(88.2 \%, \mathrm{n}$ $=284$ ) some of them dried it before consumption in order to destroy any microorganisms that might be present $(4.0 \%, \mathrm{n}=13)$; "I bake my soil in the oven first to kill all the germs", says one participant. On the other hand, $6.2 \%(n=20)$ of the consumers claimed that they washed their soil and another 1.6\% $(n=5)$ rubbed the outer layer before consumption. Knowledge of the fact that soil may contain harmful substances made these consumers attempt to "clean" their soil before ingesting it.

\section{Discussion}

Preliminary studies carried out by George and Ndip2 in rural South Africa showed a higher prevalence of $75 \%$ of women consuming soil. The lower prevalence of the study at hand indicates that geophagia may be more prevalent in rural areas compared to urban areas. All the 323 women consuming soil who visited Dr. George Mukhari Academic Hospital where the study was carried out were from surrounding peri -urban areas. The age range of 15 - 44 years shows that the habit is considered acceptable for most pregnant women and cuts across various age groups. A total of $94.8 \%(n=306)$ of the consumers had either passed through secondary or tertiary education and is indicative that literacy played no key role in the consumption of soil. This also shows that these consumers were literate enough to understand some of the health dangers associated with soil consumption.

One key reason of soil craving due to smell is in line with George and Ndip's2 studies which alluded to the conclusion that the smell and texture of the soil made it attractive to the consumers. In a separate study carried out by Nwafor17 among pregnant women at Mecklenburg Hospital, 78\% of pregnant women said that they consume soil due to its taste. The findings also indicated that some consumers exhibited other forms of pica, apart from geophagia. Roselle's18 and Crosby's19 works argued that laundry powder was often used by people practicing pica when soil was not available. In one incidence of this study, however, a consumer believed that the constipation they get from eating soil helped them to cleanse their gastro intestinal tract.

African Health Sciences Vol 16 Issue 4, December, 2016
According to Eastwell20, men have been reported to look down upon the habit of eating soil. This was in line with the current study where the responses stipulated that the behavior is somewhat secretive among the consumers and suggests that partners fairly play a key role in geophagia among pregnant women. In an independent study carried out by van Eijk et al.21 on the prevalence of geohelminth infections among pregnant women, the results indicated that married women were at a lower risk of contracting the infections. Although their study showed that there was little association between geophagia and geohelminth infections, it was evident that marital status contributed to pregnant women's health issues.

Studies carried out among the Digo community in Kenya suggested that the habit of soil eating may be picked up from parents, especially among young girls ${ }^{22}$ and the results obtained from the present survey conform to this as more consumers had a history of geophagia in their family. The high number of unemployed consumers $(73.4 \%$, $\mathrm{n}=237)$ strongly suggests that poverty plays a key role in the practice of eating soil. $5.6 \%(n=18)$ of the unemployed consumers cited the reason of pushing time during the day as there was nothing much to keep them busy.

Constipation among the consumers was classified as none, rare and frequent and when asked about the frequency of constipation, $46.4 \%(n=150)$ of the consumers reported to have had no constipation during the period of consumption. On the other hand, $35.3 \%(n=114)$ had rare constipation, while $18.3 \%(\mathrm{n}=59)$ suffered from frequent constipation. The frequency of constipation was determined by the amount of soil consumed per day as well as the frequency of consumption per day, where the more soil and the more frequent one ingested per day, the higher the chances of getting constipation. However, some consumers had experienced constipation due to the large soil amount eaten, and curbed the problem by drinking lots of water after eating. Moreover, when asked about the frequency of constipation, one frequently constipated consumer responded by saying she believed the constipation she got was the soil's way of cleaning her gut. This was an indication that constipation was considered as a health benefit, rather than a threat by some of these consumers.

The results also indicated that a good proportion of the women preferred brick red soil and this is in line with pre- 
liminary studies which established that the chief reason for geophagia, especially among pregnant women was to supplement iron supplies, and therefore fine red clay was preferred as it was believed to contain high amounts of iron which most of the consumers believe they are lacking when they eat the soil23. The women also indicated that the notion that soil contained iron was often passed on from one generation to the next within their cultures. On relating the constipation levels with the type of soil consumed, it was found that frequent constipation was common among the brown soil consumers compared to the other soil types. This could be associated with the soil structure, where the brown soil was mainly consumed as clay blocks. The black soil was considered to cause the least constipation among the soil types $(0.6 \%$ rare and $0.0 \%$ frequent constipation). This soil was mostly collected from gardens and flower pots, and thus had a larger amount of organic matter. This in turn would mean the soil had less clay material in it to cause constipation in comparison with the others.

The findings showed that geophagia was practiced by a considerable number of pregnant women attending the ante - natal clinic at Dr. George Mukhari Academic Hospital. Influences from the consumers' partners and direct family members such as the mother and grandmother acted as factors responsible for the manifestation of this practice. Poverty also played a key role as most consumers were unemployed. The habit was also found to cut across the literacy platform as it was practiced by both educated and uneducated women alike. Greater vigilance may thus be needed as part of the antenatal care classes to avoid potentially harmful health effects of the habit.

\section{Limitations}

One major challenge faced during the study was that some of the participants may have not been truthful about their soil eating habits, especially since the interviews were carried out in a hospital setting where they may have not been truthful whether or not they were geophagic in the fear of getting into trouble with health professionals. This may have compromised the findings of the study.

\section{Recommendations}

The results revealed that more than half of the women vising the ante natal clinic were soil consumers; it may be beneficial to educate the women in their earlystages of pregnancy on the dangers of soil consumption. In addition, follow up cohort studies can be done on the consumers to check whether they were only ingesting soil during pregnancy or have continued after giving birth. This can assist health professionals in ascertaining whether geophagia is linked only topregnancy or needs to beaddressed even during breastfeeding or early motherhood.

\section{References}

1. Young SL: Pica in pregnancy: new ideas about old condition. Annu Rev Nutr 2010, 30:403- 422. PubMed

2. George G, Ndip E: Prevalence of geophagia and implications to health - A study in rural South Africa. 2nd ICESD 2011 IPCBEE; 4: 166 - 169.

3. McLaughlin IJ: The Picas. Br J Hosp Med. 1987; 37: $286-290$.

4. Clark JD. Geophagy and Kalambo Falls clays. Vol3 In Kalambo Falls Prehistoric Site, The Earlier Cultures: Middle and Earlier Stone Age, Clark J. D, Ed; Cambridge University Press; Cambridge; 2001.

5. Izugbara CO. The Cultural context of geophagy among pregnant and lactating Ngwa women of south eastern Nigeria. Am. Anthropol 2003; 10: 180-199.

6. Pliny. Natural History, Rackham H, transl. London: Heinemann 1972; 9: 285

7. Setz EZF, Enzweiler J, Solferini VN, Amendola MP, Berton RS: Geophagy in the golden-faced sakimonkey (Pitheciapitheciachrysocephala) in the Central Amazon. J Zool Soc London 1999; 277: 91 - 103.

8. Krishnamani R, Mahaney WC: Geophagy among primates; Adaptive significance and ecological consequences, Anim. Behav. 2000; 59: 899 - 915

9. Young SL, Sherman PW, Lucks JB, Pelto GH: Why on earth?: Evaluating hypotheses about the physiological functions of human geophagy. Q Rev Biol. 2011; 86: 97 120.

10. Hyslop NS: Pica in man and animals. Brit J Haematol. 1977; 37: $154-155$.

11. Slamova R, Trickova M, Vonduskova H, Zraly Z, Pavlik I: Clay minerals in animal nutrition. Appl Clay Sci 2011; 51: $395-398$.

12. Starks PTB, Slabach BL: The scoop on eating dirt. Sci Am. 2012; 5: 30 - 32.

13. Mogongoa LF, Brand CE, De Jager L, Ekosse GE: Haematological and iron status of Qwaqwa women in South Africa who ingest clays. Med Tech SA 2011; 25: 33 $-37$.

14. Rose EA, Porcerelli JH, Neale AV: Pica: common but commonly missed. J Am Board Fam Med; 2000; 13: 353 358.

15. Saathof E, Olsen A, Kvalsvig JD, Gessler PW: GeoAfrican Health Sciences Vol 16 Issue 4, December, 2016 
phagy and its association with geohelminth infection in rural school children from northern KwaZulu Natal, South Africa. Trans R Soc Trop Med Hyg. 2002; 96: 485 490.

16. Walker RL: Applied Qualitative Research 1975. Gower, Aldershot; 1975.

17. Nwafor AO: Reasons pregnant women who attend ante natal care in Mecklenburg Hospital eat soil. MMed, University of Limpopo, Medunsa Campus Garankuwa Pretoria, August 2008.

18. Roselle HA: Association of laundry starch and clay ingestion with anemia in New York City, Arab Int Med, 1970; 125, 57-61 PubMed.

19. Crosby WH: When friends and patients ask about. Pica, JAMA 1976, 235: 2765.
20. Eastwell H: A pica epidemic: a price for sedentarism among Australian ex-hunter-gatherers. Psych

21. Van Eijk AM, Lindblade KA, Odhiambo F, Peterson E, Rosen DH: Geohelminth Infections among Pregnant Women in Rural Western Kenya; a Cross-Sectional Study. PLoS Neglected Tropical Diseases. 2009; 3(1): 370.

22. Kinyua F: The causes and effects of pica habit of pregnant women in Msambweni Division, Kwale District, Coast Province. Kenyatta University Repository. Pica (pathology) - Kenya, Kwale, msambweni//Pregnancy--Nutritional aspectsm 2011. (Accessed 2013 - 02 - 18).

23. Saayers G, Lipschite DA, Sayers M, Seftel HC, Bothwell TH, Charlton RW: Relationship between pica and iron nutrition in Johannesburg black adults. SA Med J; 1974; 68: 1655 - 1660 . 